Article

\title{
Isolation and Characterization of Two Klebsiella pneumoniae Phages Encoding Divergent Depolymerases
}

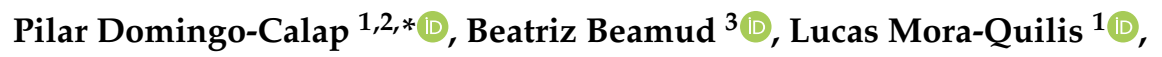 \\ Fernando González-Candelas ${ }^{1,3,4}$ (D) and Rafael Sanjuán ${ }^{1}$ (D) \\ 1 Institute for Integrative Systems Biology, I²SysBio, Universitat de València-CSIC, 46980 Paterna, Spain; \\ lucas.mora@uv.es (L.M.-Q.); fernando.gonzalez@uv.es (F.G.-C.); rafael.sanjuan@uv.es (R.S.) \\ 2 Department of Genetics, Universitat de València, 46980 Paterna, Spain \\ 3 FISABIO-Salud Pública, Generalitat Valenciana, 46020 Valencia, Spain; beatriz.beamud@uv.es \\ 4 CIBER in Epidemiology and Public Health, 46020 Valencia, Spain \\ * Correspondence: domingocalap@gmail.com; Tel.: +34-963-543-261
}

Received: 24 March 2020; Accepted: 26 April 2020; Published: 30 April 2020

check for updates

\begin{abstract}
The emergence of multidrug-resistant bacteria is a major global health concern. The search for new therapies has brought bacteriophages into the spotlight, and new phages are being described as possible therapeutic agents. Among the bacteria that are most extensively resistant to current antibiotics is Klebsiella pneumoniae, whose hypervariable extracellular capsule makes treatment particularly difficult. Here, we describe two new K. pneumoniae phages, $\pi V L C 5$ and $\pi V L C 6$, isolated from environmental samples. These phages belong to the genus Drulisvirus within the family Podoviridae. Both phages encode a similar tail spike protein with putative depolymerase activity, which is shared among other related phages and probably determines their ability to specifically infect K. pneumoniae capsular types K22 and K37. In addition, we found that phage $\pi V L C 6$ also infects capsular type K13 and is capable of striping the capsules of K. pneumoniae KL2 and KL3, although the phage was not infectious in these two strains. Genome sequence analysis suggested that the extended tropism of phage $\pi V L C 6$ is conferred by a second, divergent depolymerase. Phage $\pi V L C 5$ encodes yet another putative depolymerase, but we found no activity of this phage against capsular types other than K22 and K37, after testing a panel of 77 reference strains. Overall, our results confirm that most phages productively infected one or few Klebsiella capsular types. This constitutes an important challenge for clinical applications.
\end{abstract}

Keywords: Klebsiella pneumoniae; bacteriophage; phage therapy; wide infection range

\section{Introduction}

Klebsiella pneumoniae is an encapsulated Gram-negative bacterium that can be found as a free-living organism, in natural environments. In addition, K. pneumoniae is an opportunistic pathogen causing infections mainly in the lungs and urinary tract, although meningitis and sepsis can occur in severe cases. Due to extensive antibiotic use and favorable transmission opportunities, multidrug-resistant K. pneumoniae strains, particularly carbapenem-resistant strains [1,2], have spread in hospitals and the community. K. pneumoniae belongs to the ESKAPE priority group defined by the WHO, which also includes Enterococcus faecium, Staphylococcus aureus, Acinetobacter baumannii, Pseudomonas aeruginosa, and Enterobacter spp. [3]. Thus, K. pneumoniae is considered to be an important target for the development of new therapies.

Bacteriophages (phages) provide an interesting therapeutic alternative, since they infect bacteria in a specific manner, avoiding dysbiosis [4]. However, in the case of highly variable bacteria such as 
K. pneumoniae, the use of phages becomes challenging. It has been proposed that phage infectivity is strongly determined by the bacterial capsular type [5], making it difficult to treat a wide range of K. pneumoniae strains, since most of the phages described so far are specific to one or few capsular types. Based on this, the use of phage cocktails has been suggested, in which the interactions between phages can generate synergies that improve bacterial lysis and limit the emergence of resistances [6].

Due to the high heterogeneity of K. pneumoniae, it often becomes difficult to determine the virulence factors of this species [7]. Nevertheless, the capsule of K. pneumoniae is one such virulence factor, and hypervirulence has been associated with differences in the expression of genes involved in the capsule, or with modified capsule phenotypes [8]. Most K. pneumoniae phages have been found to encode depolymerases, which are enzymes capable of digesting the exopolysaccharide capsule by cleaving glycosidic linkages $[9,10]$. Disruption or capsule loss makes bacteria susceptible to phage infection or antibiotic treatment, suggesting a therapeutic potential for phage depolymerases [5,11-14]. Although most described phages encode a single depolymerase and, as a result, infect one or few capsular types, some phages have been reported to encode multiple depolymerases. To date, the most salient case is that of phage ØK64-1, which encodes nine different depolymerases [15]. Overall, a limited number of enzymes in active particular capsular types have been described [16]. In addition to their potential therapeutic applications, phage-borne depolymerases have been suggested for capsular typing [17]. For these reasons, discovering new Klebsiella phage depolymerases against distinct capsular types is an interesting topic with potential biomedical applications.

Recently, our group has isolated and characterized four new phages of K. pneumoniae ( $\pi V L C 1$ to $\pi V L C 4)$ from environmental samples in Valencia, Spain [18]. All of these were closely related phylogenetically, belonged to the genus Drulisvirus, encoded only one depolymerase, and showed a very narrow capsular type range. Using a similar protocol, here we isolated two new phages with high lytic activity, which we named $\pi V L C 5$ and $\pi V L C 6$. As opposed to our previously described phages, these new isolates were capable of infecting several reference capsular types of Klebsiella spp. Whole genome analysis revealed that $\pi V L C 5$ and $\pi V L C 6$ encoded divergent depolymerases, potentially explaining their host tropisms.

\section{Results}

\subsection{Isolation and Phenotypic Characterization of Two New Klebsiella Pneumoniae Phages}

Phage $\pi V L C 5$ was obtained from a water sample in an irrigation ditch near a wastewater treatment plant, whereas phage $\pi V L C 6$ was isolated from a river, near its outflow to the sea, in an area where waste discharged by nearby villages accumulates. Our initial screening was carried out using a multidrug-resistant clinical isolate of K. pneumoniae as host, which belongs to capsular type K22. Once plaques were observed, they were further isolated and plaque-purified. Amplification of the phages in liquid medium was carried out to obtain a high-titer lysate and to proceed with detailed phage characterization. Plaque assays showed that the amplified phage $\pi V L C 5$ reached a titer of $2.4 \times 10^{11}$ plaque forming units (PFU) per $\mathrm{mL}$, while $\pi V L C 6$ reached $1.0 \times 10^{10} \mathrm{PFU} / \mathrm{mL}$. For both phages, large plaques were observed, which were surrounded by a halo that is often interpreted as an indicator of depolymerase-mediated digestion of bacterial capsules (Figure 1a). Haloes are formed because depolymerases can diffuse and degrade capsules in nearby bacteria even if lysis does not occur in these areas. This has been previously shown in other Klebsiella spp. [18] and in phages infecting other encapsulated bacteria, such as Escherichia coli or Acinetobacter baumannii [19,20]. The haloes of phage $\pi V L C 6$ were wider than those of $\pi V L C 5$, whereas lysed areas were larger for $\pi V L C 5$, suggesting that these were two distinct phages. Transmission electron microscopy micrographs obtained from high-titer lysates showed that the virion morphology of the two phages was similar and typical of podoviruses, with an icosahedral head of approximately $50 \mathrm{~nm}$ and a small tail of about $10 \mathrm{~nm}$ (Figure 1b). 
(a)
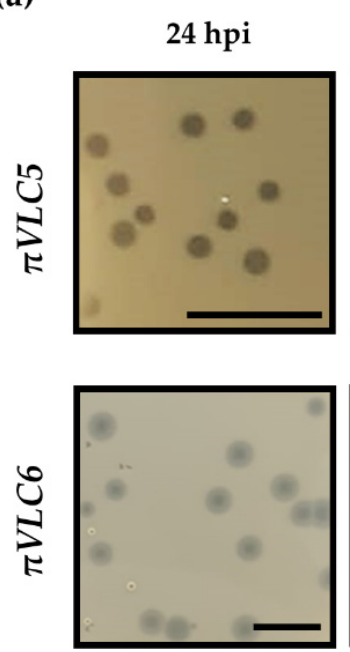

48 hpi
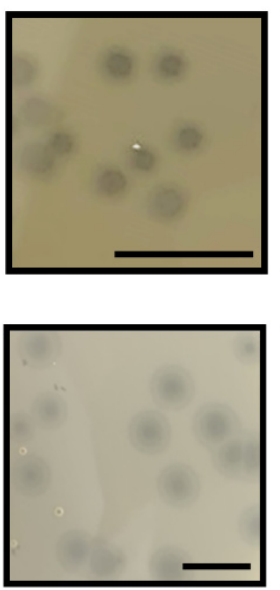

72 hpi
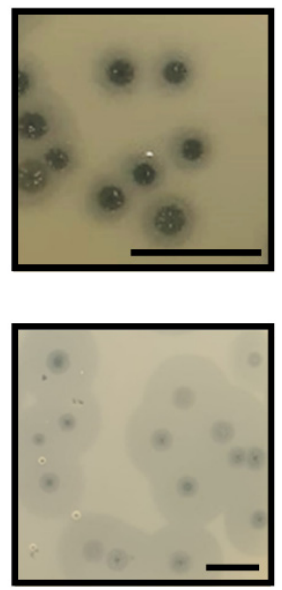

(b)

\section{$\pi V L C 5$}

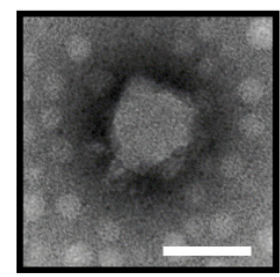

$\pi V L C 6$

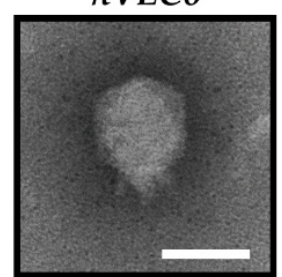

Figure 1. Initial characterization of Klebsiella phages $\pi V L C 5$ and $\pi V L C 6$. (a) Plaque assays of the two phages. Plaques were allowed to develop in soft agar media at $37^{\circ} \mathrm{C}$ (hpi: hours post inoculation). Notice that haloes around plaques kept expanding even though the lysis did not proceed further, suggesting passive diffusion of depolymerase activity. Scale bar: $1 \mathrm{~cm}$. (b) Transmission electron micrographs of the two novel K. pneumoniae phages. Scale bar: $50 \mathrm{~nm}$.

\subsection{Genome Sequencing and Comparative Genomics}

Genome sequencing of $\pi V L C 5$ yielded a single contig of $44,932 \mathrm{bp}(54.3 \% \mathrm{G}+\mathrm{C}$ content) containing 58 putative coding sequences (CDSs). For phage $\pi V L C 6$, a single contig was also obtained with 44,294 bp (54.2\% G+C content) and 60 CDSs. The two phages showed a pairwise nucleotide identity of $92.5 \%$. BLAST analysis showed that the two phages belonged to the family Podoviridae, genus Drulisvirus. To verify this, we obtained a multiple sequence alignment of all publicly available classified drulisviruses $(n=9)$, including the four additional genomes recently published by our group [18]. Klebsiella phages $\pi V L C 5$ and $\pi V L C 6$ were found to be collinear and similar to the rest of drulisviruses. To determine the evolutionary relationships among these phages, maximum likelihood (ML) phylogenies of whole-genome and RNA polymerase sequences were built. The whole-genome tree showed that Klebsiella phages $\pi V L C 5$ and $\pi V L C 6$ were members of a well-supported cluster within drulisviruses, but that $\pi$ VLC5 was closer to the Klebsiella phage Vb_KpnP_SU552A [21] (Figure 2a). According to this phylogeny, both phages were phylogenetically separated from the previously described $\pi V L C 1-4$ phages. Yet, the RNA polymerase tree was not consistent with the whole genome tree and suggested a closer relationship between $\pi V L C 5$ and the previously described phages $\pi V L C 1$ and $\pi V L C 2$ (Figure 2b). 
(a)

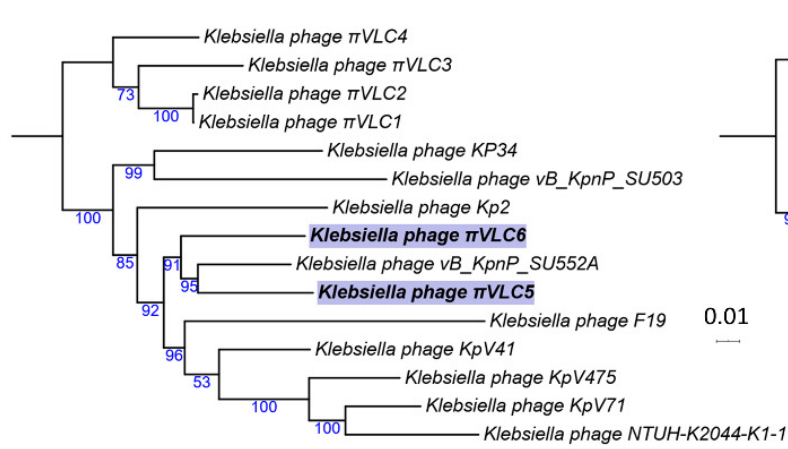

(b)

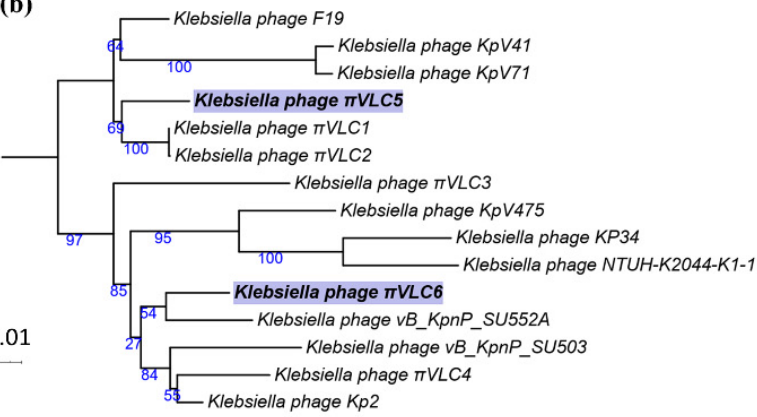

Figure 2. Maximum likelihood (ML) phylogenetic tree of the Drulisvirus genus. (a) Whole-genome and (b) RNA polymerase. Numbers indicate bootstrap support values (1000 pseudo-replicates). The two new phages are highlighted in purple. The scale bar represents 0.01 sequence divergence (percent nucleotide substitutions).

\subsection{Functional Annotation of K. pneumoniae Phages $\pi V L C 5$ and $\pi V L C 6$}

Twenty-four conserved proteins were found among drulisviruses, including Klebsiella phages $\pi V L C 5$ and $\pi V L C 6$. These included proteins involved in DNA replication (DNA polymerase and DNA-dependent RNA polymerase, 5'-3' exonuclease, helicase, and primase), DNA packaging (DNA maturase $\mathrm{A}$ and $\mathrm{B}$ ), head proteins (tegument, collar, capsid, internal virion, and core proteins), tail tubular proteins (A and B), enzymatic activities (phosphoesterase and peptidase), lysis proteins (spanin and holin), and additional, hypothetical proteins. Most differences were observed in the tail fiber proteins, where similarity dropped drastically between phages $\pi V L C 5, \pi V L C 6$, and the rest of drulisviruses, with a few exceptions (Figure 3, Table S1). Both phages contained two putative tail fiber proteins, with the second located after the lysis cassette, as previously observed [22].

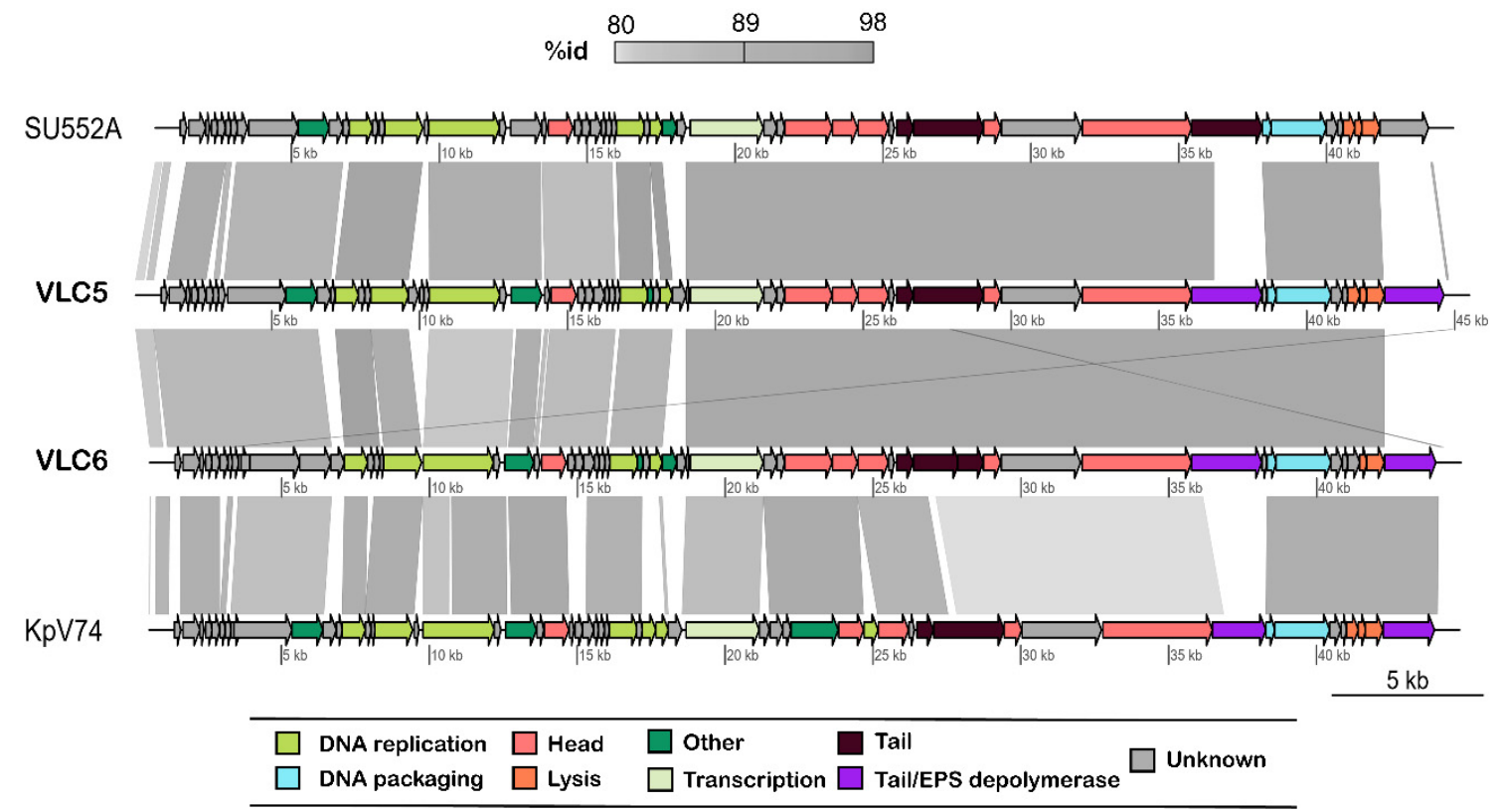

Figure 3. Genome alignment of the Klebsiella phages $\pi V L C 5$ and $\pi V L C 6$ with the previously characterized Klebsiella phages $v B \_K p n P \_S U 552 A$ (closest relative) and $v B \_K p n P \_K p V 74$. Arrows represent coding sequences (CDSs). The different shades of gray represent different identity levels. The figure was obtained using genoPlotR [23].

The first tail-fiber protein was completely shared between the $\pi V L C 5$ and $\pi V L C 6$ phages (ORF49 and ORF51, respectively) and had a high structural similarity to the tail spike protein of bacteriophage 
phiAB6 (e-value $3.9 \times 10^{-33}$ ). Hence, we annotated both ORFs as tail spikes. In addition, these two ORFs showed high sequence similarity with the tail fiber protein of the Pantoea bacteriophage LIMElight (e-values $6.0 \times 10^{-40}$ and $4.0 \times 10^{-40}$, respectively), available from the depolymerases database. These ORFs contained two sequence domains. Positions 13-140 matched significantly with bacteriophage T7 tail fiber protein, whereas positions 308-610 matched with a pectin lyase domain (Table 1), where the depolymerase activity is expected to reside.

Table 1. Depolymerase motifs identified in Klebsiella phages $\pi V L C 5$ and $\pi V L C 6$.

\begin{tabular}{|c|c|c|c|c|c|c|c|}
\hline Phage & ORF & $\begin{array}{c}\text { Protein } \\
\text { Size (aa) }\end{array}$ & Tool & Motif (aa) & Family & Identifier & e-Value \\
\hline \multirow{8}{*}{$\pi V L C 5$} & \multirow{4}{*}{$\begin{array}{c}\text { ORF49 } \\
(36103-38478)\end{array}$} & \multirow{4}{*}{792} & \multirow[t]{2}{*}{ InterProScan5 } & $13-140$ & $\begin{array}{l}\text { Bacteriophage } T 7 \\
\text { tail fiber protein }\end{array}$ & IPR005604 & $5.1 \times 10^{-14}$ \\
\hline & & & & $319-617$ & Pectin lyase & IPR012334 & $3.2 \times 10^{-10}$ \\
\hline & & & \multirow{2}{*}{ HHpred } & $2-578$ & phiAB6 tail spike & 5JSD_B & $2.1 \times 10^{-23}$ \\
\hline & & & & $234-789$ & $\begin{array}{l}\text { Putative tail fiber; } \\
\text { Tail spike, hydrolase }\end{array}$ & 5W6S_A & $2.3 \times 10^{-13}$ \\
\hline & \multirow{4}{*}{$\begin{array}{c}\text { ORF58 } \\
(42631-44634)\end{array}$} & \multirow{4}{*}{668} & \multirow{3}{*}{ InterProScan5 } & $16-365$ & Pectin lyase & IPR012334 & $1.0 \times 10^{-15}$ \\
\hline & & & & $544-603$ & $\begin{array}{l}\text { Chaperone of } \\
\text { endosialidase }\end{array}$ & IPR030392 & $7.3 \times 10^{-37}$ \\
\hline & & & & $544-667$ & $\begin{array}{l}\text { Winged helix-like } \\
\text { DNA-binding } \\
\text { domain }\end{array}$ & IPR036388 & $5.8 \times 10^{-6}$ \\
\hline & & & HHpred & $26-665$ & $\begin{array}{l}\text { Tail spike protein } \\
\text { Acinetobacter phage }\end{array}$ & 6EU4_B & $6.0 \times 10^{-34}$ \\
\hline \multirow{4}{*}{$\pi V L C 6$} & \multirow{3}{*}{$\begin{array}{c}\text { ORF51 } \\
(35765-38140)\end{array}$} & \multirow{3}{*}{792} & \multirow[t]{2}{*}{ InterProScan5 } & $13-140$ & $\begin{array}{l}\text { Bacteriophage } T 7 \\
\text { tail fiber protein }\end{array}$ & IPR005604 & $1.7 \times 10^{-14}$ \\
\hline & & & & $319-617$ & Pectin lyase & IPR012334 & $9.1 \times 10^{-10}$ \\
\hline & & & HHpred & $1-578$ & phiAB6 tail spike & 5JSD_B & $6.3 \times 10^{-23}$ \\
\hline & $\begin{array}{c}\text { ORF58 } \\
(42292-44025)\end{array}$ & 578 & HHpred & $1-576$ & $\begin{array}{l}\text { Bacteriophage } \\
\text { CBA120 tail spike }\end{array}$ & 6EU4_B & $2.1 \times 10^{-41}$ \\
\hline
\end{tabular}

Phage $\pi V L C 5$ encoded a second tail fiber/spike protein (ORF58) that also matched significantly with the sequences in the depolymerase database, the best match being with the tail fiber protein of the Klebsiella phage KP32 (e-value $1.4 \times 10^{-55}$ ). InterProScan5 revealed three different domains in this ORF-a pectin lyase, an endosialidase chaperone, and a DNA-binding domain. In this case, the closest protein structure determined by HHpred was the tail spike of an Acinetobacter phage (Table 1). In turn, the second tail fiber protein of phage $\pi V L C 6$ (ORF58) was similar to a putative pectate lyase of Serratia phage phiMAM1. Surprisingly, no protein domains were found with InterProScan 5 for this ORF. However, the ORF matched significantly with the protein structure of bacteriophage CBA120 tail spike (Table 1).

\subsection{Divergence of Putative Depolymerase Sequences of Klebsiella Phages $\pi V L C 5$ and $\pi V L C 6$}

To elucidate the degree of divergence and find putative orthologs for the different tail fibers/spikes of phages $\pi V L C 5$ and $\pi V L C 6$, the above four ORFs were extracted and searched against a custom phage database with BLASTN. The tail fiber/spike shared among $\pi V L C 5$ and $\pi V L C 6$ (ORF49 and ORF51) was also present in the rest of Drulisvirus analyzed (Figure 4), including non-Klebsiella phages. However, only the first domain was highly conserved, whereas the second domain (where the putative enzymatic activity resides) was absent in all of these sequences except for the $\pi V L C$ phages and partly in Klebsiella phage KP32 (Table S1). The common region of the tail fiber was not restricted to this genus. In fact, it was similar to the tail fibers of Przondovirus and Phikmvoirus phages, all in the Autographivirinae subfamily (Table S1). Remarkably, the average nucleotide identity values dropped in this region, ranging from $70.7 \%$ to $99.8 \%$, when compared to the rest of the genome (from $89.5 \%$ to 
$99.8 \%$ ). Therefore, this protein seemed to be unique to the $\pi V L C$ phages, given that the other phages only showed small regions of limited sequence identity. However, the ML phylogenetic tree of the core region of this ORF did not group the novel phages $\pi V L C 5$ and $\pi V L C 6$, with the rest of $\pi V L C$ phages (Figure 4).

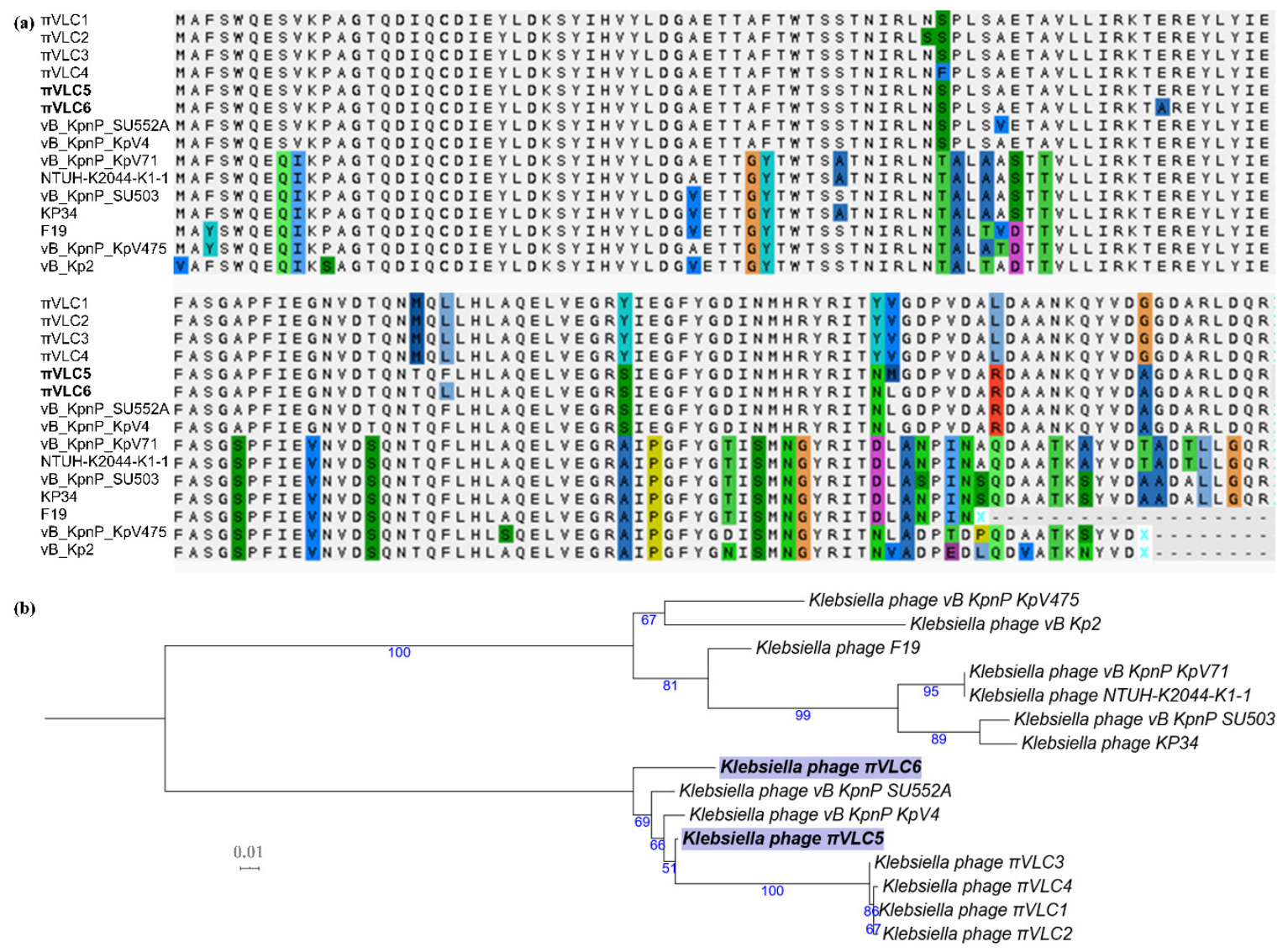

Figure 4. Conserved tail fibers/spikes of available drulisviruses (a). Amino acid sequence alignment of the conserved region of the tail fiber/spike. Variant amino acids are highlighted in different colors. (b). Nucleotide ML tree of the conserved region of the tail fiber/spike. Numbers indicate bootstrap values (1000 pseudo-replicates). The scale bar represents 0.01 sequence divergence (percent nucleotide substitutions).

Regarding the additional tail fiber/spikes of $\pi V L C 5$ and $\pi V L C 6$, only three significant hits were found for the phage $\pi$ VLC5 ORF58 (Table S1). The best hit was a hypothetical protein of the Klebsiella phage KOX1 with a sequence coverage of $51 \%$ and $68 \%$ nucleotide identity. This hit contained the pectin lyase domain. The second hit was the tail fiber protein of the Klebsiella phage NJS1 (13\% coverage and $67 \%$ identity). Klebsiella phages KOX1 and NJS1 belonged to the genus Webervirus of the family Siphoviridae. The last hit was almost non-significant (only $2 \%$ coverage but $89 \%$ of identity), with a protein of the Vibrio phage 1.164.O. For phage $\pi V L C 6$ ORF58, we found three hits that covered the entire ORF, all from bacteriophages of the same genus, with levels of identity ranging from $94.8 \%$ to 95.7\% (Table S1).

\subsection{Determination of the Host Range of the Two Klebsiella Pneumoniae Phages $\pi V L C 5$ and $\pi V L C 6$}

A panel of 77 reference strains of Klebsiella spp. purchased from the Statens Serum Institute (Copenhagen, Denmark) was used to test the infectivity of the newly discovered phages using spot tests in semi-solid medium and OD measurements in liquid cultures. Overall, the results of the spot tests were consistent with those obtained in liquid infections (Figure 5, Table S2). Phage $\pi V L C 5$ had a 
lytic ability against capsular types K22 and K37, whereas phage $\pi V L C 6$ lysed capsular types K22, K37, and $\mathrm{K} 13$ capsular types. Liquid cultures also showed that, for K22 and K37, phage-resistant bacteria emerged at around $5 \mathrm{~h}$ post-inoculation (hpi) and eventually reached densities similar to those of uninfected cultures at approximately $10 \mathrm{hpi}$, a process that could not be prevented using a cocktail of the two phages. In K13, $\pi V L C 6$-mediated bacterial lysis occurred less efficiently but, on the other hand, the OD values remained lower than those of uninfected cultures even at 12 hpi. For K2 and, to a lesser extent, K3, spot tests revealed that phage $\pi V L C 6$ produced a slight reduction in the turbidity of the bacterial lawns. Liquid infections also indicated a slight growth delay in K2 cultures inoculated with $\pi V L C 6$.

(a)

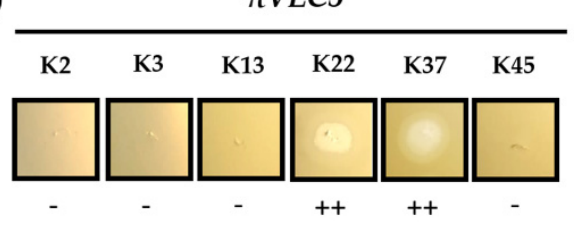

$\pi V L C 6$

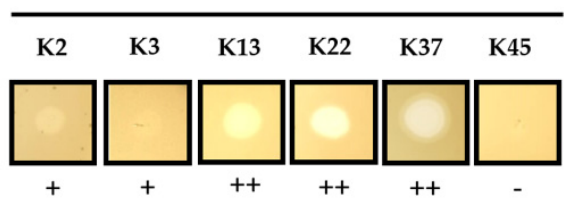

(b)
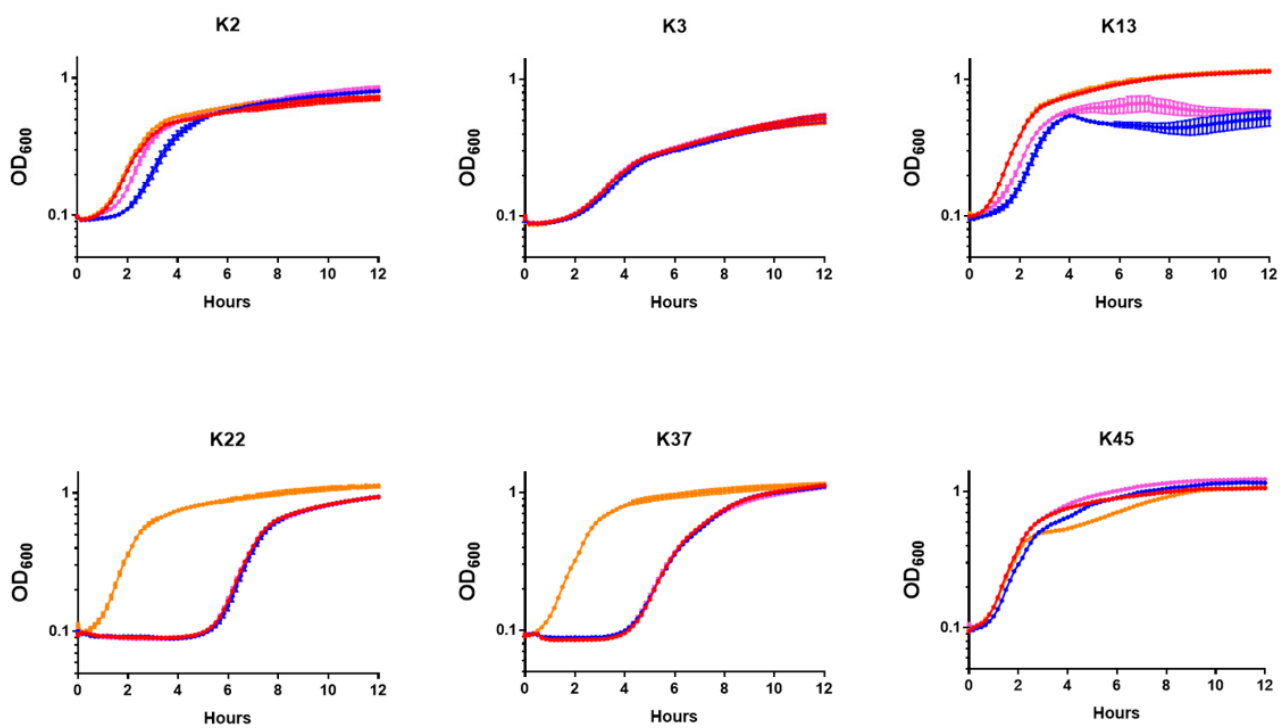

Figure 5. Lytic activity of Klebsiella phages $\pi V L C 5$ and $\pi V L C 6$ in different capsular types. (a) Spot tests of the two K. pneumoniae phages in K2, K3, K13, K22, K37, and K45 Klebsiella sp. reference strains. All 77 reference strains were assayed, but only those showing phage activity are shown, except for K45, which is included as an example of a non-susceptible strain. Plaques were allowed to develop in soft agar media overnight at $37^{\circ} \mathrm{C} .++$ : obvious lysis; +: halo-like turbidity reduction; -: no effect. (b) OD measurements of capsular types K2, K3, K13, K22, K37, and K45 Klebsiella spp. Orange-non-infected control; red- $\pi V L C 5$; blue $-\pi V L C 6$; pink $-\pi V L C 5+\pi V L C 6$. Error bars indicate the SEM.

In bacterial strains where spot tests revealed an effect of the phage, phage progeny production was evaluated by inoculating low-density cultures (OD ca. 0.05 ) with ca. $10^{5} \mathrm{PFU}$ (as determined in K22 plaque assays). At $4 \mathrm{hpi}$, phage titration showed that $\pi V L C 5$ and $\pi V L C 6$ did not produce any progeny in $\mathrm{K} 2$ and $\mathrm{K} 3$ cultures, since the endpoint titers were similar or lower than those at inoculation. The absence of phage progeny was also verified in semi-solid cultures inoculated by the spot test method. In contrast, K22 and K37 were productively infected by both phages, yielding $>10^{8} \mathrm{PFU} / \mathrm{mL}$ of $\pi V L C 5$ and $>10^{9} \mathrm{PFU} / \mathrm{mL}$ of $\pi V L C 6$. In addition, phage $\pi V L C 6$ was able to produce $>10^{9} \mathrm{PFU} / \mathrm{mL}$ in $\mathrm{K} 13$, as opposed to phage $\pi V L C 5$. 


\subsection{Depolymerase Activity of Phage $\pi V L C 6$}

Phage $\pi V L C 6$ did not infect the reference strains of capsular types $\mathrm{K} 2$ and $\mathrm{K} 3$, as indicated by the absence of phage progeny. However, $\pi V L C 6$ did produce a halo in K3 lawns (and less obviously, also in K2 lawns) and slightly retarded the growth of K2 cultures. We hypothesized that this effect might be due to the ability of a $\pi V L C 6$ depolymerase to digest the K2 and K3 capsules. This depolymerase activity could be encoded by ORF58, which showed no homologous gene in $\pi V L C 5$. To test this, we sampled the halo formed by $\pi V L C 6$ in K2 and K3 lawns for microscopy. For comparison, we also analyzed regions of the plate where no phage was added. These samples were stained with crystal violet to visualize bacteria and with nigrosin to provide background contrast. In non-inoculated K2 and $\mathrm{K} 3$ bacteria, capsules were intact and easily observable as non-stained areas around each cell (Figure 6). In contrast, capsules were obviously absent in K2 and K3 bacteria taken from the halo regions of the cultures inoculated with phage $\pi V L C 6$. Our results confirmed the degradation of the exopolysaccharide capsule by the action of a depolymerase encoded by $\pi V L C 6$, despite the inability of the phage to infect and lyse these strains.

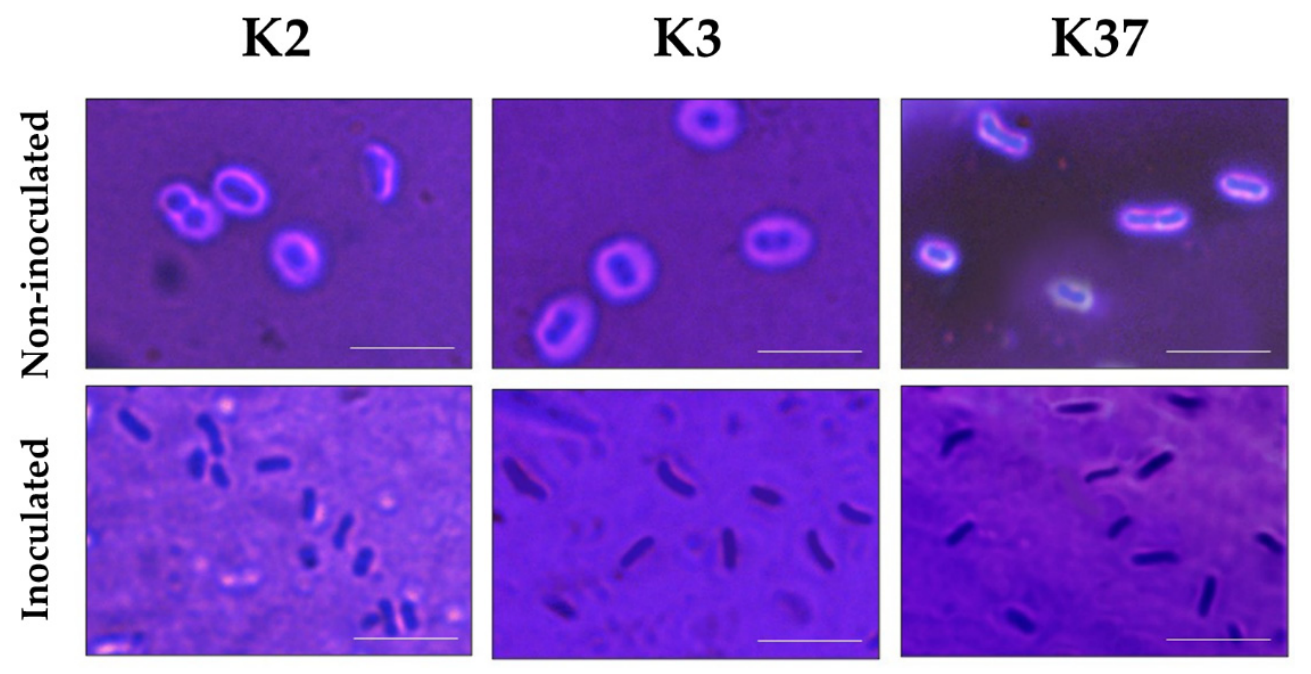

Figure 6. Klebsiella phage $\pi V L C 6$ depolymerase activity against K2 and K3 strains. Cultures were doubly stained with nigrosin and crystal violet. Capsules appeared as non-stained areas around cells. Inoculation with phage $\pi V L C 6$ produced a nearly complete stripping of the capsules. K37 strain was used as a positive control. Scale bar: $5 \mu \mathrm{m}$.

\subsection{Structural Comparison of K Antigens}

Host-range experiments revealed that both phages $\pi V L C 5$ and $\pi V L C 6$ were able to infect capsular types K22 and K37. Additionally, phage $\pi V L C 6$ could infect $\mathrm{K} 13$ and digest $\mathrm{K} 2$ and $\mathrm{K} 3$ capsules. As depolymerases are thought to be antigenically specific, we wanted to determine whether these five capsular types shared some structural backbone that could make them recognizable by common enzymes. The K. pneumoniae capsule is a polysaccharide matrix of repeated units with an acidic component. K22 and K37 have the same sequence of sugars, with the exception of the presence of OAc substituents in K22 [24]. Approximately $66 \%$ of the atoms of K22 and K37 align properly, with low values of RMSD $(0.115 \AA$ ) as expected (Figure 7). K2 and K13 shared the units of glucose, mannose, and glucuronic acid $[25,26]$. This could be observed in the overlapping of 36 atoms in the structural pairwise alignment with an RMSD value of $0.025 \AA$. The RMSD values between these two capsular types, $\mathrm{K} 2$ and $\mathrm{K} 13$, and those described above, $\mathrm{K} 22$ and $\mathrm{K} 37$, were much higher (>2.5 $\mathrm{\AA}$ ), showing a poor overlap of their molecular structures. Interestingly, antigen $\mathrm{K} 3$ overlapped partially with both clusters (K22/K37 and K2/K13), with RMSD ranging from $0.049 \AA$ to $0.110 \AA$. The repeated units of the $\mathrm{K} 3$ structure were made of pyruvic acetal-bearing pentasaccharide containing galactose, mannose, and glucuronic acid [27]. Thus, K3 shared the sugars mannose with K2/K13 and galactose with K22/K37. 
K2 vs. K13

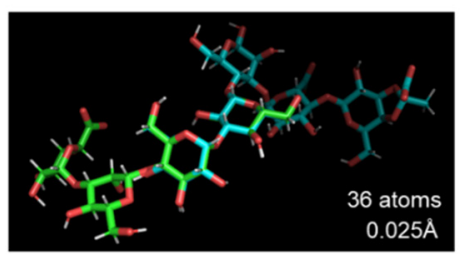

K22 vs. K37

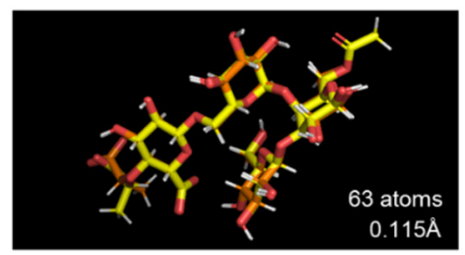

K2, K13 vs K3

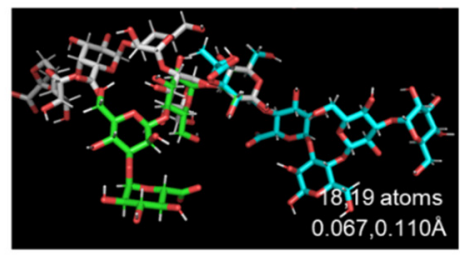

K22, K37 vs. K3

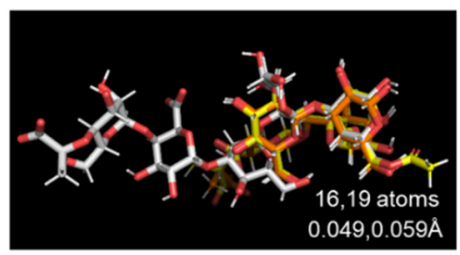

K2 vs. K22,K37

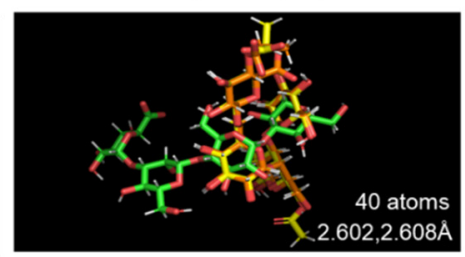

K22, K37 vs. K13

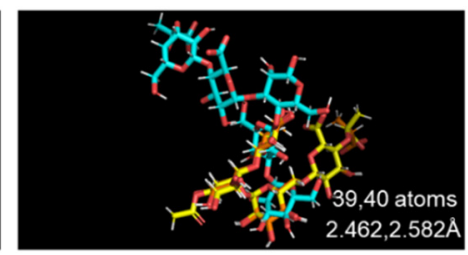

Figure 7. Structural superposition of Klebsiella $\mathrm{K}$ antigens $\mathrm{K} 2$, K3, K13, K22, and K37. The number of atoms that align between the pair-wise structures and the RMSD values are indicated. Green-K2 (85 atoms), white-K3 (112 atoms), blue-K13 (112 atoms), yellow-K22 (98 atoms), and orange-K37 (93 atoms).

\section{Discussion}

The K. pneumoniae exopolysaccharide capsule is a major virulence factor and a source of diversity that complicates treatment [28]. Still relatively little is known about the diversity of Klebsiella sp. phages and the molecular mechanisms allowing infection of different capsular types. Although previous studies have proposed that depolymerases play a key role in the recognition and entry of phages in these bacteria, additional data are needed to establish more general patterns. Recently, our group has phenotypically and molecularly characterized four new Drulisviruses infecting capsular type K22 [18]. Despite the fact that this capsular type is not highly prevalent, it has been found among multi-drug-resistant clinical isolates. Moreover, Klebsiella capsular diversity is widely distributed, meaning that no single capsular type monopolizes a large fraction of clinical isolates and that, consequently, it would be necessary to target most types for efficiently combating this pathogen. Here, we have characterized two new phages capable of infecting not only K22, but also other capsular types.

Phylogenetic analyses of whole and core genomes of the genus Drulisvirus group the new phages together, along with the previously described Klebsiella phage $V b \_K p n P \_S U 552 A$. However, the phylogeny of the RNA polymerase does not support this grouping, placing $\pi V L C 5$ with other $\pi V L C$ phages. These results, taken together with the lack of homology in one of the tail spikes, suggest a certain degree of mosaic-like genome structure for drulisviruses. Horizontal acquisition of tail spike genes seems especially relevant in the case of ORF58 of the Klebsiella phage $\pi V L C 5$ and ORF58 of $\pi V L C 6$. The former presents a unique structure among the phage sequences described so far, as it is only distantly related to other phages from a different family. On the contrary, the second tail spike of Klebsiella phage $\pi V L C 6$ is also present in four other phages of the same genus, with a high level of identity. Regarding the shared tail spike present in all members of the genus Drulisvirus and some related phages (Przondovirus and Phikmvvirus), the enzymatic domain is only found in the $\pi V L C$ phages, all of which share the ability to infect K22 capsular types. Interestingly, phage $\pi V L C 5$ was sampled in the same place as phages $\pi V L C 1$ and $\pi V L C 2$, and these three phages seemed to form a monophyletic group according to the RNA polymerase phylogeny. However, they differed in their depolymerase domains and whole-genome phylogenetic position. This suggests that gene exchange events were involved in adaption to specific ecological niches [22].

Both phages described were capable of lysing capsular types K22 and K37. In addition, in both cases, lysis haloes were observed around plaques, presumably resulting from the action of their depolymerases [29]. Klebsiella strains presenting capsular types K22 and K37 shared most of the genes involved in the synthesis of the capsule polysaccharides, except for one gene that showed a 
frameshift mutation [30] and the overall biochemical structure as explained above. This could explain why both phages, $\pi$ VLC5 and $\pi$ VLC6, were able to infect these two capsular types. In principle, the depolymerase shared between these two phages (and the phages previously described by our group) should be responsible for degrading the exopolysaccharides shared between K22 and K37. The ability of the phage $\pi \mathrm{VLC} 6$ to also infect capsular type K13 and digest $\mathrm{K} 2$ capsule should be conferred by its second depolymerase, due to the divergence shown between the capsular biochemical structures. This second putative depolymerase presented high nucleotide identity ( $96 \%$ and $100 \%$ of coverage) with the second tail-fiber protein of bacteriophage $v B \_K p n P \_K p V 74$ [16]. Solovieva et al., cloned and expressed this protein (kpv74_56, APZ82768.1) and showed that it was a functional depolymerase against K2 strains and one K13 strain, thus, sharing not only the nucleotide identity but also host tropism with the second depolymerase of bacteriophage $\pi V L C 6$ [22]. The association between $\mathrm{K} 2$ and K13 capsule digestion was also supported by previous work showing cross-infectivity between the K2 and $\mathrm{K} 13$ types [31]. The additional interaction of phage $\pi V L C 6$ with the $\mathrm{K} 3$ strain was less expected, as the biochemical structure of this antigen overlapped only partially with $\mathrm{K} 22 / \mathrm{K} 37$ and $\mathrm{K} 2 / \mathrm{K} 13$, so both enzymes might be able to recognize the K3 antigen. However, there are no reported cases of cross-reaction involving these antigens. To our knowledge, the only depolymerase confirmed to have $\mathrm{K} 3$ tropism is that of the Klebsiella phage KP32, KP32gp37 [5]. Interestingly, this protein is the only one described so far that presents the identity of the enzymatic domain of the first tail spike shared by all $\pi V L C$ phages (Table S1). Thus, the diversity of this enzymatic domain as well as the role of the second tail spike should be further explored, in order to explain K3 tropism. Lastly, we did not find any additional host specificity of $\pi V L C 5$, despite the presence of a highly divergent depolymerase. Screening against additional novel capsular types, which are constantly being described [32], might reveal its role.

In conclusion, the two new phages $\pi V L C 5$ and $\pi V L C 6$, showed interesting aspects of the Drulisvirus evolution and suggest that host range expansion can occur via the acquisition of divergent depolymerases. Yet, few broad-range Klebsiella phages have been described so far. Further prospection of environmental samples might reveal new, more broadly-acting phages. In future work, it would also be interesting to explore resistance mechanisms, since in most cases bacterial growth fully relapses after few hours. In vitro resistance conferred by capsule loss might be inconsequential in vivo, since de-capsulated mutants tend to be avirulent [33]. Finally, testing synergistic effects between phages and antibiotics is yet another promising line of research [34,35].

\section{Materials and Methods}

\subsection{Bacteria}

The clinical isolate 1210 obtained from Hospital La Fe (Valencia, Spain) was used as a host to test environmental samples for phage discovery. In addition, 77 reference strains of Klebsiella spp. purchased from the Statens Serum Institute (Copenhagen, Denmark; Table S3) were used to determine the range of infection of the isolated phages.

\subsection{Isolation of Phages from Environmental Samples}

A water sample obtained from a river and another from an irrigation ditch were used to isolate phages. For this purpose, $50 \mathrm{~mL}$ of water were taken and kept at room temperature until processing in the laboratory, typically $1 \mathrm{~h}$ later. After vortexing, samples were centrifuged $(4000 \times g, 10 \mathrm{~min})$ and supernatants filtered through $0.45 \mu \mathrm{m}$ and $0.22 \mu \mathrm{m}$, to remove the cells and debris. Then, $1 \mathrm{~mL}$ of the filtered samples was added to $500 \mu \mathrm{L}$ of K. pneumoniae clinical isolate 1210 and plated on Petri dishes, through the top agar method. Plates were incubated at $37^{\circ} \mathrm{C}$ for $20 \mathrm{~h}$. Isolated plaques of different morphology were then recovered by aspiration with a micropipette and stored at $-70{ }^{\circ} \mathrm{C}$. In order to check the isolated plaques and purify them, two additional plaque assays and plaque picking steps were performed. The plaque-purified phages were amplified in liquid media by infecting an 
exponential growth phase culture of the bacterium $\left(\mathrm{OD}_{600}=0.4\right)$. After lysis, bacteria and debris were removed by centrifugation $(13,000 \times g, 3 \mathrm{~min}$ twice) and the supernatants were aliquoted and stored at $-70^{\circ} \mathrm{C}$.

\subsection{Electron Microscopy}

High titer lysates were centrifuged and supernatants were filtered through $0.22 \mu \mathrm{m}$ to remove bacteria and debris. A drop from each lysate was then deposited onto a carbon-coated Formvar supported by a 300 mesh copper grid. After air drying for $30 \mathrm{~min}$, excess liquid was removed with filter paper. Phages were negatively stained with $2 \%$ phosphotungstic acid and examined under an electron microscope Jeol JEM-1010.

\subsection{DNA Isolation and Genome Sequencing}

DNA was extracted by treating high titer filtered lysates with DNAse I to remove non-encapsidated DNA and, following DNAse I inactivation, DNA extraction was performed using a commercial kit (QIAamp, QIAGEN). DNA was then fragmented by sonication and sequenced in an Illumina MiSeq machine yielding 250 paired-end reads, which were screened for contaminants using Kraken 2 [36] and then assembled with SPAdes v3.9.1 [37]. Contigs smaller than 1000 nucleotides were discarded, resulting in a single contig for each genome. PHASTER [38] and BLAST were used to determine the closest sequences in the databases. These were downloaded, and we used ProgressiveMAUVE [39] to obtain a whole-genome multiple sequence alignment. Complete genome sequences are available at Genbank (accession No. MT197175-MT197176).

\subsection{Genome Annotation}

Phages were annotated using PHANOTATE [40], Glimmer v.3.0 [41], and Prodigal [42]. Gene callings and start and stop coordinates were compared with a custom script available at https://github.com/BBeamud/. Nucleotide sequences for the putative ORFs were extracted with seqtk subseq (https://github.com/lh3/seqtk) and used for functional annotation. A phage nucleotide database was built with makeblastdb from the one available at http://millardlab.org/bioinformatics/ bacteriophage-genomes/. This database contains all non-redundant phage proteins sequences deposited in GenBank and their annotation (August, 2019). Phages $\pi V L C$ were added to this database [18]. The best BLASTN hit of non-hypothetical proteins, when possible, was retrieved with an e-value cutoff of $10^{-5}$. We also searched for temperate behavior in our phage genomes, such as mobile genetic elements, antibiotic resistance or virulence genes, and any kind of bacterial gene. Local databases from ICEberg v.2.0 (http://db-mml.sjtu.edu.cn/ICEberg/), the Comprehensive Antibiotic Resistance Database (CARD, https://card.mcmaster.ca/), Virulence factors of Pathogenic Bacteria (VFDB, http://www.mgc.ac.cn/VFs/main.htm), and BacteriaDB (in-house database, 2016) were used to accomplish this using BLASTN, with an e-value cutoff of $10^{-5}$. Lastly, CDSs were searched against a local database constructed with depolymerase proteins included in Pires et al. [43]. InterProScan5 [44] and HHpred [45] were used for further checking of domains and protein structures when a significant similarity was found.

\subsection{Comparative Genomics}

Whole-genome average nucleotide identity (ANI) values between the new Klebsiella phages and the other Drulisvirus phages were obtained using pANIto (https://github.com/sanger-pathogens/panito). Conserved and unique genes among the Drulisvirus were obtained with Proteinortho v.6 [46], by comparing the previously extracted nucleotide CDSs and the ones available from GenBank. Maximum likelihood (ML) phylogenies were constructed with 1000 fast bootstrap pseudo-replicates using the GTR+G+I substitution model in IQ-TREE v.1.6.5 [47]. 


\subsection{Determination of Host Range}

Spot tests were performed by adding $2 \mu \mathrm{L}$ drops of each high titer phage to the bacterial lawns of the 77 reference Klebsiella spp. strains in top-agar semi-solidified media, and incubating plates at $37^{\circ} \mathrm{C}$ for $24 \mathrm{~h}$. In addition, we inoculated cultures of the 77 reference strains in w- 96 plates with $10^{5} \mathrm{PFU}$ of phage and incubated cultures at $37^{\circ} \mathrm{C}$ in a plate reader (Multiskan) for $10 \mathrm{~h}$, to determine the strength of lysis based OD measurements taken every $10 \mathrm{~min}$.

\subsection{Determination of Phage Progeny Production}

Approximately $10^{5} \mathrm{PFU}$ were used to inoculate $500 \mu \mathrm{L}$ of log-phase bacterial cultures $\left(\mathrm{OD}_{600}=0.05\right)$ in a Thermoshaker, and the cultures were incubated at $250 \mathrm{rpm}$ and $37^{\circ} \mathrm{C}$. At $4 \mathrm{hpi}$, the cultures were centrifuged $(13,000 \times \mathrm{g}, 2 \mathrm{~min})$ to remove bacteria and debris, and the supernatants were used for titration. To accomplish this, the supernatants were serially diluted in LB, and then used to perform plaque assays in $1210 \mathrm{~K}$. pneumoniae cultures. A similar approach was used to determine phage progeny production in semi-solid cultures. Spots with ca. $10^{5} \mathrm{PFU}$ were used to inoculate bacterial cultures, that were incubated at $37^{\circ} \mathrm{C}$ for $8 \mathrm{~h}$. After this, a micropipette was used to recover the phages from the spot, which were resuspended in LB for titration.

\subsection{Capsule Light Microscopy}

Bacteria were grown in semi-solidified media in petri dishes to perform spot tests with phage suspensions $\left(\geq 10^{7} \mathrm{PFU} / \mathrm{mL}\right)$. After $24 \mathrm{~h}$ at $37^{\circ} \mathrm{C}$, a smear loop was used to sample bacteria from the haloes and non-inoculated regions of the plate. Samples were fixed with $2.5 \%$ formaldehyde for $20 \mathrm{~min}$, in the presence of $100 \mathrm{mM}$ lysine, which prevented capsule loss during fixation [48]. After removing the fixative agent through centrifugation and washing with low-salt TE buffer, $10 \%$ nigrosin was mixed with the bacterial suspension on a glass slide and $1 \%$ crystal violet was added. The preparations were examined through a light microscopy and the presence of capsules was assessed by the exclusion of both nigrosin and crystal violet around the cells.

\subsection{Structural Comparison of K Antigens}

To determine if the capsular $\mathrm{K}$ antigens that showed any type of interaction with the novel phages were structurally similar, we downloaded the atomic structure of each antigen from https://iith.ac.in/K-PAM/k_antigen.html [49]. Program database (PDB) files were loaded and aligned with the PyMOL Molecular Graphics System (Version 2.0 Schrödinger, LLC). The 'align' command performed a structural superposition with several rounds of refinement to discard structural outliers. We obtained the number of aligned atoms and the root-mean-square deviation of atomic positions (RMSD) between the pairwise-alignments of $\mathrm{K}$ antigens.

Supplementary Materials: The following are available online at http://www.mdpi.com/1422-0067/21/9/3160/s1. Table S1: Results of the BLASTN search of the tail fiber sequences for phages $\pi V L C 5$ and $\pi V L C 6$ against a database of phage CDSs. Table S2: Determination of Klebsiella phages $\pi V L C 5$ and $\pi V L C 6$ host range in semi-solid and liquid. Table S3: Klebsiella species and K-serotypes of the 77 K-type reference strains from the Statens Serum Institute (Copenhagen, Denmark).

Author Contributions: Conceptualization, P.D.-C. and R.S.; Funding acquisition, P.D.-C., F.G.-C., and R.S.; Investigation, P.D.-C., B.B., and L.M.-Q.; Methodology, P.D.-C., B.B., L.M.-Q., and R.S.; Supervision, P.D.-C., F.G.-C., and R.S.; Writing-original draft, P.D.-C. and B.B.; Writing-review and editing, P.D.-C., F.G.-C., and R.S. All authors have read and agreed to the published version of the manuscript.

Funding: This research was funded by the ESCMID Research Grant 20200063 to P.D-C., ERC Consolidator Grant 724519 Vis-a-Vis to R.S, and project BFU2017-89594R from MICINN to F.G-C. B.B was funded by a PhD fellowship from Spanish MCIU FPU16/02139.

Acknowledgments: We thank Carles Úbeda for the clinical K. pneumoniae 1210 strain, Justine Vienne for help with phage hunting, and María Duran for technical assistance.

Conflicts of Interest: The authors declare no conflict of interest. 


\section{References}

1. Holt, K.E.; Wertheim, H.; Zadoks, R.N.; Baker, S.; Whitehouse, C.A.; Dance, D.; Jenney, A.; Connor, T.R.; Hsu, L.; Severin, J.; et al. Genomic analysis of diversity, population structure, virulence, and antimicrobial resistance in Klebsiella pneumoniae, an urgent threat to public health. Proc. Natl. Acad. Sci. USA 2015, 112, E3574-E3581. [CrossRef] [PubMed]

2. Effah, C.Y.; Sun, T.; Liu, S.; Wu, Y. Klebsiella pneumoniae: An increasing threat to public health. Ann. Clin. Microbiol. Antimicrob. 2020, 19, 1-9. [CrossRef] [PubMed]

3. Founou, R.C.; Founou, L.L.; Essack, S.Y. Clinical and economic impact of antibiotic resistance in developing countries: A systematic review and meta-analysis. PLoS ONE 2017, 12, e0189621. [CrossRef] [PubMed]

4. Domingo-Calap, P.; Georgel, P.; Bahram, S. Back to the future: Bacteriophages as promising therapeutic tools. HLA 2016, 87, 133-140. [CrossRef] [PubMed]

5. Majkowska-Skrobek, G.; Latka, A.; Berisio, R.; Squeglia, F.; Maciejewska, B.; Briers, Y.; Drulis-Kawa, Z. Phage-Borne Depolymerases Decrease Klebsiella pneumoniae Resistance to Innate Defense Mechanisms. Front. Microbiol. 2018, 9, 2517. [CrossRef]

6. Kortright, K.E.; Chan, B.K.; Koff, J.L.; Turner, P.E. Phage Therapy: A Renewed Approach to Combat Antibiotic-Resistant Bacteria. Cell Host Microbe 2019, 25, 219-232. [CrossRef]

7. Imai, K.; Ishibashi, N.; Kodana, M.; Tarumoto, N.; Sakai, J.; Kawamura, T.; Takeuchi, S.; Taji, Y.; Ebihara, Y.; Ikebuchi, K.; et al. Clinical characteristics in blood stream infections caused by Klebsiella pneumoniae, Klebsiella variicola, and Klebsiella quasipneumoniae: A comparative study, Japan, 2014-2017. BMC Infect. Dis. 2019, 19, 946. [CrossRef]

8. Palacios, M.; Miner, T.A.; Frederick, D.R.; Sepúlveda, V.; Quinn, J.D.; Walker, K.A.; Miller, V.L. Identification of Two Regulators of Virulence That Are Conserved inKlebsiella pneumonia Classical and Hypervirulent Strains. mBio 2018, 9, e01443-18. [CrossRef]

9. Adams, M.H.; Park, B.H. An enzyme produced by a phage-host cell system. Virology 1956, 2, 719-736. [CrossRef]

10. Rieger-Hug, D.; Stirm, S. Comparative study of host capsule depolymerases associated with Klebsiella bacteriophages. Virology 1981, 113, 363-378. [CrossRef]

11. Lin, T.-L.; Hsieh, P.-F.; Huang, Y.-T.; Lee, W.-C.; Tsai, Y.-T.; Su, P.-A.; Pan, Y.-J.; Hsu, C.-R.; Wu, M.-C.; Wang, J.-T. Isolation of a Bacteriophage and Its Depolymerase Specific for K1 Capsule of Klebsiella pneumoniae: Implication in Typing and Treatment. J. Infect. Dis. 2014, 210, 1734-1744. [CrossRef] [PubMed]

12. Majkowska-Skrobek, G.; Latka, A.; Berisio, R.; Maciejewska, B.; Squeglia, F.; Romano, M.; Lavigne, R.; Struve, C.; Drulis-Kawa, Z. Capsule-Targeting Depolymerase, Derived from Klebsiella KP36 Phage, as a Tool for the Development of Anti-Virulent Strategy. Viruses 2016, 8, 324. [CrossRef] [PubMed]

13. Hsieh, P.-F.; Lin, H.-H.; Lin, T.-L.; Chen, Y.-Y.; Wang, J.-T. Two T7-like Bacteriophages, K5-2 and K5-4, Each Encodes Two Capsule Depolymerases: Isolation and Functional Characterization. Sci. Rep. 2017, 7, 4624. [CrossRef] [PubMed]

14. Wu, Y.; Wang, R.; Xu, M.; Liu, Y.; Zhu, X.; Qiu, J.; Liu, Q.; He, P.; Li, Q. A Novel Polysaccharide Depolymerase Encoded by the Phage SH-KP152226 Confers Specific Activity Against Multidrug-Resistant Klebsiella pneumoniae via Biofilm Degradation. Front. Microbiol. 2019, 10, 2768. [CrossRef] [PubMed]

15. Pan, Y.-J.; Lin, T.-L.; Chen, C.-C.; Tsai, Y.-T.; Cheng, Y.-H.; Chen, Y.-Y.; Hsieh, P.-F.; Lin, Y.-T.; Wang, J.-T. Klebsiella Phage $\Phi$ K64-1 Encodes Multiple Depolymerases for Multiple Host Capsular Types. J. Virol. 2017, 91, e02457-16. [CrossRef]

16. Pan, Y.-J.; Lin, T.-L.; Chen, Y.-Y.; Lai, P.-H.; Tsai, Y.-T.; Hsu, C.-R.; Hsieh, P.-F.; Lin, Y.-T.; Wang, J.-T. Identification of three podoviruses infecting Klebsiella encoding capsule depolymerases that digest specific capsular types. Microb. Biotechnol. 2019, 12, 472-486. [CrossRef]

17. Knecht, L.E.; Veljkovic, M.; Fieseler, L. Diversity and Function of Phage Encoded Depolymerases. Front. Microbiol. 2020, 10, 2949. [CrossRef]

18. Domingo-Calap, P.; Beamud, B.; Vienne, J.; González-Candelas, F.; Sanjuán, R. Isolation of Four Lytic Phages Infecting Klebsiella pneumoniae K22 Clinical Isolates from Spain. Int. J. Mol. Sci. 2020, 21, 425. [CrossRef]

19. Morales, A.C.H.; Lessor, L.L.; Wood, T.L.; Migl, D.; Mijalis, E.M.; Cahill, J.; Russell, W.K.; Young, R.; Gill, J.J. Genomic and Biochemical Characterization of Acinetobacter Podophage Petty Reveals a Novel Lysis Mechanism and Tail-Associated Depolymerase Activity. J. Virol. 2018, 92, e01064-17. [CrossRef] 
20. Mushtaq, N.; Redpath, M.B.; Luzio, J.P.; Taylor, P.W. Treatment of experimental Escherichia coli infection with recombinant bacteriophage-derived capsule depolymerase. J. Antimicrob. Chemother. 2005, 56, 160-165. [CrossRef]

21. Eriksson, H.; Maciejewska, B.; Latka, A.; Majkowska-Skrobek, G.; Hellstrand, M.; Melefors, Ö.; Wang, J.-T.; Kropinski, A.M.; Drulis-Kawa, Z.; Nilsson, A. A Suggested New Bacteriophage Genus, "Kp34likevirus", within the Autographivirinae Subfamily of Podoviridae. Viruses 2015, 7, 1804-1822. [CrossRef] [PubMed]

22. Solovieva, E.V.; Myakinina, V.P.; Kislichkina, A.A.; Krasilnikova, V.M.; Verevkin, V.V.; Mochalov, V.V.; Lev, A.; Fursova, N.K.; Volozhantsev, N.V. Comparative genome analysis of novel Podoviruses lytic for hypermucoviscous Klebsiella pneumoniae of K1, K2, and K57 capsular types. Virus Res. 2018, 243, 10-18. [CrossRef] [PubMed]

23. Guy, L.; Kultima, J.R.; Andersson, S.G.E. genoPlotR: Comparative gene and genome visualization in R. Bioinformatics 2010, 26, 2334-2335. [CrossRef] [PubMed]

24. Parolis, L.A.; Parolis, H.; Niemann, H.; Stirm, S. Primary structure of Klebsiella serotype K22 capsular polysaccharide: Another glycan containing 4-O-[(S-1-carboxyethyl]-d-glucuronic acid. Carbohydr. Res. 1988, 179, 301-314. [CrossRef]

25. Sutherland, I.W. The Exopolysaccharides of Klebsiella Serotype 2 Strains as Substrates for Phage-induced Polysaccharide Depolymerases. J. Gen. Microbiol. 1972, 70, 331-338. [CrossRef]

26. Niemann, H.; Frank, N.; Stirm, S. Klebsiella serotype-13 capsular polysaccharide: Primary structure and depolymerization by a bacteriophage-borne glycanase. Carbohydr. Res. 1977, 59, 165-177. [CrossRef]

27. Dutton, G.G.; Parolis, H.; Joseleau, J.-P.; Marais, M.-F. The use of bacteriophage depolymerization in the structural investigation of the capsular polysaccharide from Klebsiella serotype K3. Carbohydr. Res. 1986, 149, 411-423. [CrossRef]

28. Paczosa, M.K.; Mecsas, J. Klebsiella pneumoniae: Going on the Offense with a Strong Defense. Microbiol. Mol. Biol. Rev. 2016, 80, 629-661. [CrossRef]

29. Sutherland, I.W.; Hughes, K.A.; Skillman, L.C.; Tait, K. The interaction of phage and biofilms. FEMS Microbiol. Lett. 2004, 232, 1-6. [CrossRef]

30. Pan, Y.-J.; Lin, T.-L.; Chen, Y.-H.; Hsu, C.-R.; Hsieh, P.-F.; Wu, M.-C.; Wang, J.-T. Capsular Types of Klebsiella pneumoniae Revisited by wzc Sequencing. PLoS ONE 2013, 8, e80670. [CrossRef]

31. Pieroni, P.; Rennie, R.P.; Ziola, B.; Deneer, H.G. The use of bacteriophages to differentiate serologically cross-reactive isolates of Klebsiella pneumoniae. J. Med. Microbiol. 1994, 41, 423-429. [CrossRef] [PubMed]

32. Bellich, B.; Lagatolla, C.; Rizzo, R.; D'Andrea, M.M.; Rossolini, G.M.; Cescutti, P. Determination of the capsular polysaccharide structure of the Klebsiella pneumoniae ST512 representative strain KPB-1 and assignments of the glycosyltransferases functions. Int. J. Biol. Macromol. 2020, 155, 315-323. [CrossRef] [PubMed]

33. Lawlor, M.S.; Hsu, J.; Rick, P.D.; Miller, V.L. Identification of Klebsiella pneumonia virulence determinants using an intranasal infection model. Mol. Microbiol. 2005, 58, 1054-1073. [CrossRef]

34. Domingo-Calap, P.; Delgado-Martínez, J. Bacteriophages: Protagonists of a Post-Antibiotic Era. Antibiotics 2018, 7, 66. [CrossRef] [PubMed]

35. Bansal, S.; Harjai, K.; Chhibber, S. Depolymerase improves gentamicin efficacy during Klebsiella pneumoniae induced murine infection. BMC Infect. Dis. 2014, 14, 456. [CrossRef] [PubMed]

36. Wood, D.E.; Lu, J.; Langmead, B. Improved metagenomic analysis with Kraken 2. Genome Biol. 2019, $20,257$. [CrossRef] [PubMed]

37. Bankevich, A.; Nurk, S.; Antipov, D.; Gurevich, A.; Dvorkin, M.; Kulikov, A.; Lesin, V.M.; Nikolenko, S.; Pham, S.; Prjibelski, A.D.; et al. SPAdes: A New Genome Assembly Algorithm and Its Applications to Single-Cell Sequencing. J. Comput. Biol. 2012, 19, 455-477. [CrossRef]

38. Arndt, D.; Grant, J.R.; Marcu, A.; Sajed, T.; Pon, A.; Liang, Y.; Wishart, D.S. PHASTER: A better, faster version of the PHAST phage search tool. Nucleic Acids Res. 2016, 44, W16-W21. [CrossRef]

39. Darling, A.E.; Mau, B.; Perna, N.T. progressiveMauve: Multiple Genome Alignment with Gene Gain, Loss and Rearrangement. PLoS ONE 2010, 5, e11147. [CrossRef]

40. McNair, K.; Zhou, C.; Dinsdale, E.A.; Souza, B.; Edwards, R.A. PHANOTATE: A novel approach to gene identification in phage genomes. Bioinformatics 2019, 35, 4537-4542. [CrossRef]

41. Delcher, A. Improved microbial gene identification with GLIMMER. Nucleic Acids Res. 1999, $27,4636-4641$. [CrossRef] 
42. Hyatt, D.; Chen, G.; Locascio, P.F.; Land, M.; Larimer, F.W.; Hauser, L. Prodigal: Prokaryotic gene recognition and translation initiation site identification. BMC Bioinform. 2010, 11, 119. [CrossRef]

43. Pires, D.P.; Oliveira, H.; Melo, L.D.R.; Cerqueira, M.A.; Azeredo, J. Bacteriophage-encoded depolymerases: Their diversity and biotechnological applications. Appl. Microbiol. Biotechnol. 2016, 100, 2141-2151. [CrossRef]

44. Jones, P.; Binns, D.; Chang, H.Y.; Fraser, M.; Li, W.; McAnulla, C.; McWilliam, H.; Maslen, J.; Mitchell, A.L.; Nuka, G.; et al. InterProScan 5: Genome-scale protein function classification. Bioinformatics 2014, 30, 1236-1240. [CrossRef] [PubMed]

45. Zimmermann, L.; Stephens, A.; Nam, S.-Z.; Rau, D.; Kübler, J.; Lozajic, M.; Gabler, F.; Soeding, J.; Lupas, A.N.; Alva, V. A Completely Reimplemented MPI Bioinformatics Toolkit with a New HHpred Server at its Core. J. Mol. Biol. 2018, 430, 2237-2243. [CrossRef] [PubMed]

46. Lechner, M.; Findeiss, S.; Müller, L.; Marz, M.; Stadler, P.F.; Prohaska, S. Proteinortho: Detection of (Co-)orthologs in large-scale analysis. BMC Bioinform. 2011, 12, 124. [CrossRef] [PubMed]

47. Nguyen, L.-T.; Schmidt, H.A.; Von Haeseler, A.; Minh, B.Q. IQ-TREE: A Fast and Effective Stochastic Algorithm for Estimating Maximum-Likelihood Phylogenies. Mol. Biol. Evol. 2014, 32, 268-274. [CrossRef] [PubMed]

48. Jacques, M.; Graham, L. Improved preservation of bacterila capsule for electron microscopy. J. Electron Microsc. Tech. 1989, 11, 167-169. [CrossRef] [PubMed]

49. Patro, L.P.P.; Sudhakar, K.U.; Rathinavelan, T. K-PAM: A unified platform to distinguish Klebsiella species Kand O-antigen types, model antigen structures and identify hypervirulent strains. bioRxiv 2019. [CrossRef]

(C) 2020 by the authors. Licensee MDPI, Basel, Switzerland. This article is an open access article distributed under the terms and conditions of the Creative Commons Attribution (CC BY) license (http://creativecommons.org/licenses/by/4.0/). 